Disclosure Information A. Lepland: None. A. Malfanti: None. U. Haljasorg: None. S. Dordevic: None. L. Salumäe: None. P. Peterson: None. T. Teesalu: None. M.J. Vicent: None. P. Scodeller: None.

\section{P01.08 SARCOPENIA AS BIOMARKER FOR IMMUNOTHERAPY OUTCOMES AND IMMUNE-RELATED ADVERSE EVENTS - A SYSTEMATIC REVIEW AND META-ANALYSIS}

${ }^{1} S$ Günther*, ${ }^{1} \mathrm{P}$ Trinkner, ${ }^{2} \mathrm{M}$ von Bergwelt, 'D Cordas dos Santos, 'S Theurich. ' Gene Center LMU, München, Germany; ${ }^{2}$ LMU University Hospital Munich, Department of Medicine III, München, Germany

\subsection{6/jitc-2021-ITOC8.12}

Background Sarcopenia is an established risk factor for oncologic treatments like surgical interventions and conventional chemotherapy. However, the impact of sarcopenia on treatment and immune-related adverse events (irAEs) of cancer patients treated with immune checkpoint inhibitors (ICIs) continues to be debated. Therefore, we performed a systematic review and meta-analysis of all published articles evaluating the effects of sarcopenia on survival outcomes and irAEs of patients undergoing ICI treatment.

Materials and Methods In analogy to the Cochrane guidelines for systematic reviews, we performed a systematic literature search including all published articles in PubMed until February 2021 for the key terms 'sarcopenia' or 'sarcopenic obesity' in combination with several terms for ICI treatments, irrespective of cancer entity and ICI used. Further selection criteria for meta-analysis included defined cut-offs for sarcopenia. Reported outcomes included progression-free survival (PFS), overall survival (OS) and the frequency of irAEs. For the random effects meta-analysis, we used Hazard Ratios (HR) for OS and PFS and Odds Ratios (OR) for occurrence of irAEs with corresponding $95 \%$ confidence intervals $(95 \% \mathrm{CI})$, respectively.

Results A total of 15 studies with 1,428 patients were selected to be eligible for meta-analysis. To evaluate muscle mass, all studies used CT-derived body composition parameters at the third lumbar vertebrae level and defined sarcopenia by using skeletal muscle index (SMI), psoas muscle index (PMI) or skeletal muscle density (SMD). Sarcopenic patients showed an inferior survival compared to non-sarcopenic patients with a combined HR for PFS with 1.53 (95\%CI 1.23-1.91, $\mathrm{p}=$ $0.0001)$ and for OS with 1.6 (95\% CI 1.23-2.09, $\mathrm{p}=$ $0,0005)$. Frequency of irAEs did not differ between sarcopenic and non-sarcopenic patients regardless of irAE grade (irAEs of grade $\geq 3$ : OR $1.14,95 \%$ CI $0.65-2.01, \mathrm{p}=0.64$, irAEs of any grade: OR $0.96,95 \% \mathrm{CI} 0.65-1.42, \mathrm{p}=0.85$ ).

Conclusions This is the first meta-analysis that assessed sarcopenia in a mixed cohort of cancer patients. It revealed that sarcopenia is an adverse risk factor for survival of patients undergoing ICI treatment without affecting the risk of developing irAEs. Future studies may address sarcopenia as a patient-derived risk factor emphasizing the importance of nutrition and physical activity interventions.

Disclosure Information S. Günther: None. P. Trinkner: None. M. von Bergwelt: None. D. Cordas dos Santos: None. S. Theurich: None.

\section{P02 Tumor microenvironment and microbiome in Immunotherapy}

\section{P02.01 T- AND B-CELL ABUNDANCE ARE REMARKABLY REDUCED IN THE IMMUNE MICROENVIRONMENT OF POST-TRANSPLANT MALIGNANCIES}

${ }^{1,2}{ }^{2}$ Schran* ${ }^{*},{ }^{2} \mathrm{R}$ Datta, ${ }^{3} \mathrm{O}$ Persa, ${ }^{2} \mathrm{C}$ Aguilar, ${ }^{1} \mathrm{M}$ Thelen, ${ }^{1} \mathrm{~J}$ Lehmann, ${ }^{1} \mathrm{M}$ Garcia-Marquez,
${ }^{1} \mathrm{~K}$ Wennhold, ${ }^{4} \mathrm{~A}$ Quaas, ${ }^{2} \mathrm{C}$ Bruns, ${ }^{3} \mathrm{C}$ Mauch, ${ }^{4} \mathrm{H}$ Löser, ${ }^{2} \mathrm{D}$ Stippel, ${ }^{1,2} \mathrm{H}$ Schlößer.
${ }^{1}$ University Hospital of Cologne, Center for Molecular Medicine, Cologne, Germany;
${ }^{2}$ University Hospital of Cologne, Department of General, Visceral and Cancer Surgery,
Cologne, Germany; ${ }^{3}$ University Hospital of Cologne, Department of Dermatology, Cologne,
Germany; ${ }^{4}$ University Hospital of Cologne, Department of Pathology, Cologne, Germany

10.1136/jitc-2021-ITOC8.13

Background Immunosuppressive medication is mandatory in the majority of solid organ transplant recipients to reduce the risk of allograft rejection. An increased risk to develop cancer is a negative side effect of long-term immunosuppression and impaired cancer immunosurveillance is assumed as underlying mechanism. However, the impact of immunosuppression on the tumor immune microenvironment (TME) is poorly understood. In this study we aimed to elucidate differences between immune infiltrates of post-transplant malignancies and cancer of non-immunosuppressed patients.

Materials and Methods 117 resected tumor samples of 80 organ transplant (kidney, heart, lung and liver) recipients were included. Immunohistochemistry and digital image analysis of whole section slides was used to quantify T- (CD3, CD8) and B-cell (CD20) abundance in the TME of 14 different cancer types. These data were used to calculate the Immune-score and to quantify tertiary lymphoid structures in the TME. Expression of Human-Leucocyte-Antigen-I (HLA-I) and programmed cell death ligand 1 (PD-L1) were analyzed in tissue microarrays. Clinical parameters were included in statistical analyses.

Results The increased risk of cancer in organ transplant recipients was reflected by a remarkably reduced immune infiltrate in the central region (CT) and the surrounding tissue (invasive margin, IM) of cancer areas. $\mathrm{T}$ cell abundance was decreased in IM and CT of skin (814 vs. $1440 \mathrm{CD}^{+}$ cells $\left./ \mathrm{mm}^{2}, \mathrm{p}<0.01\right)$ and non-skin tumors (479 vs. 781 $\mathrm{CD}^{+}$cells $\left./ \mathrm{mm}^{2}, \mathrm{p}<0.01\right)$, when compared to non-immunosuppressed controls. These differences were more pronounced in the IM than in the CT and larger when comparing abundance of $\mathrm{CD} 8^{+} \mathrm{T}$ cells. The Immune-score integrating results from CT and IM was also decreased in transplant recipients. Similar to the results observed for $\mathrm{T}$ cells, B cell abundance and density of tertiary lymphoid structures were lower in cancer samples of transplant recipients. Decreased expression of HLA-I was more common in transplant recipients whereas the fraction of samples with PD-L1 expression was higher in controls.

Conclusions Our study demonstrates that post-transplant malignancies show a low immune infiltrate and supports the hypothesis of reduced anti-tumor immune response as an important mechanism underlying increased risk of cancer in organ transplant recipients. Optimized immunosuppressive protocols may be able to reduce cancer incidence and cancer therapies need to consider the distinct immune microenvironment of post-transplant malignancies. 
Disclosure Information S. Schran: None. R. Datta: None. O. Persa: None. C. Aguilar: None. M. Thelen: None. J. Lehmann: None. M. Garcia-Marquez: None. K. Wennhold: None. A. Quaas: None. C. Bruns: None. C. Mauch: None. H. Löser: None. D. Stippel: None. H. Schlößer: None.

\section{P02.02 SINGLE-CELL RNA SEQUENCING OF NEUROBLASTOMA TUMORS REVEALS IMMUNOREGULATORY INTERACTIONS AS NOVEL TARGETS FOR IMMUNOTHERAPY}

J Wienke*, WM Kholosy, LL Visser, KM Keller, P Lijnzaad, T Margaritis, KPS Langenberg, RR De Krijger, FCP Holstege, JJ Molenaar. Princess Máxima Center for Pediatric Oncology, Utrecht, Netherlands

\subsection{6/jitc-2021-ITOC8.14}

Background Immunotherapy with CAR-T cells, as well as immune checkpoint blockade, show limited clinical efficacy in the pediatric solid cancer neuroblastoma, despite the success in various adult cancers. The lacking efficacy may be due to various immune evasion strategies employed by neuroblastoma tumors, leading to altered functionality of tumor-infiltrating immune cells. We aimed to provide a comprehensive overview of the composition and function of the neuroblastoma immune environment, as well as relevant immunoregulatory interactions $(=)$, to identify novel targets for immunotherapy. Materials and Methods 25 tumor samples from 20 patients (17 with high-risk disease, 6 with MYCN amplification), were collected during diagnostic biopsy pre-treatment $(n=10)$ or during resection surgery after induction chemotherapy $(n=15)$. Samples were enzymatically digested, single-cell FACS sorted and sequenced by Cel-Seq2 protocol.

Results Lymphoid cells in the TME consisted of $\alpha \beta-, \gamma \delta$-T cells, NK cells and B cells. Among $\alpha \beta-\mathrm{T}$ cells we identified $\mathrm{CD}^{+} \mathrm{T}$ cells, two functionally distinct clusters of $\mathrm{CD}^{+} \mathrm{T}$ cells, naive-like $\mathrm{T}$ cells and $\mathrm{FOXP}^{+}$regulatory $\mathrm{T}$ cells (Tregs). $\mathrm{CD}^{+} \mathrm{T}$ cells had reduced cytotoxic capacity compared to blood-derived $\mathrm{T}$ cells from a reference group. Tregs expressed high levels of PRDM1, LAYN and ICOS, suggesting an effector Treg profile, which is associated with increased inhibitory capacity. Although NK cells expressed the cytotoxic genes NKG7, KLRF1, GNLY, GZMB and PRF1, their expression was significantly lower than in blood-derived reference NK cells. Gene set enrichment analysis (GSEA) confirmed a reduced cytotoxic capacity of tumoral NK cells, which correlated with a decreased expression of activating receptors $(\mathrm{r}=0.41$, $\mathrm{p}<0.001)$ and increased TGF $\beta$ signaling $(\mathrm{r}=-0.45, \mathrm{p}<0.001)$. In addition, NK cells highly expressed the heterodimeric receptor KLRC1:KLRD1, which can inhibit NK cell function through HLA-E binding. High HLA-E expression by endothelial, immune and mesenchymal cells confirmed its inhibitory activity in the TME. Within the myeloid compartment we identified various immunosuppressive populations, comprising a cluster of IL10 and VEGFA expressing macrophages, three clusters of M2 differentiated macrophages expressing MMP9 and LGALS3, and dendritic cells with intact antigen presenting capacity, but high expression of numerous genes encoding immunosuppressive molecules such as IDO1, LGALS1, LGALS2, CCL22 and NECTIN2. In MYCN amplified tumors, specifically, we observed even lower cytotoxic capacity of $\mathrm{CD}^{+} \mathrm{T}$ and NK cells. We identified increased TGFB1 expression and defective antigen presentation by myeloid and tumor cells as potential causes for reduced cytotoxicity in MYCN amplified tumors. To identify relevant targets for immunotherapy we constructed an unbiased interaction network, which revealed NECTIN1 $=C D 96$ and $M I F=C D 74$ as active immunoregulatory interactions between tumor and T/NK cells, and CD80/CD86=CTLA4, CLEC2D $=$ KLRB1, HLA-E $=$ KLRC1/ KLRC2, CD99=PILRA, LGALS9=HAVCR2, and NECTIN2 =TIGIT between myeloid and T/NK cells.

Conclusions Cytotoxic lymphocytes in the neuroblastoma TME show reduced cytotoxic capacity, likely due to highly immunosuppressive myeloid cells, Tregs and numerous immunoregulatory interactions, which may serve as novel targets for immunotherapy in neuroblastoma.

Disclosure Information J. Wienke: None. W.M. Kholosy: None. L.L. Visser: None. K.M. Keller: None. P. Lijnzaad: None. T. Margaritis: None. K.P.S. Langenberg: None. R.R. De Krijger: None. F.C.P. Holstege: None. J.J. Molenaar: None.

\section{P02.03 AUTOMATED CELL TYPE SPECIFIC PD-L1 QUANTIFICATION BY ARTIFICIAL INTELLIGENCE USING HIGH THROUGHPUT BLEACH \& STAIN 15-MARKER MULTIPLEX FLUORESCENCE IMMUNOHISTOCHEMISTRY IN HUMAN CANCERS}

NC Blessin*, E Bady, T Mandelkow, C Yang, J Raedler, R Simon, C Fraune, M Lennartz, S Minner, E Burandt, D Höflmayer, G Sauter, SA Weidemann. University Medical Center Hamburg-Eppendorf, Hamburg, Germany

\subsection{6/jitc-2021-ITOC8.15}

Background The quantification of PD-L1 (programmed cell death ligand 1) has been used to predict patient's survival, to characterize the tumor immune microenvironment, and to predict response to immune checkpoint therapies. However, a framework to assess the PD-L1 status with a high interobserver reproducibility on tumor cells and different types of immune cells has yet to be established.

Materials and Methods To study the impact of PD-L1 expression on the tumor immune microenvironment and patient outcome, a framework for fully automated PD-L1 quantification on tumor cells and immune cells was established and validated. Automated PD-L1 quantification was facilitated by incorporating three different deep learning steps for the analysis of more than 80 different neoplasms from more than 10 '000 tumor specimens using a bleach \& stain 15-marker multiplex fluorescence immunohistochemistry panel (i.e., PDL1, PD-1, CTLA-4, panCK, CD68, CD163, CD11c, iNOS, CD3, CD8, CD4, FOXP3, CD20, Ki67, CD31). Clinicopathological parameter were available for more than 30 tumor entities and overall survival data were available for 1517 breast cancer specimens.

Results Comparing the automated deep-learning based PD-L1 quantification with conventional brightfield PD-L1 data revealed a high concordance in tumor cells $(\mathrm{p}<0.0001)$ as well as immune cells $(p<0.0001)$ and an accuracy of the automated PD-L1 quantification ranging from $90 \%$ to 95.2\%. Across all tumor entities, the PD-L1 expression level was significantly higher in distinct macrophage/dendritic cell (DC) subsets (identified by CD68, CD163, CD11c, iNOS; $\mathrm{p}<000.1)$ and in macrophages/DCs located in the Stroma $(p<0.0001)$ as compared to intratumoral macrophages/DC subsets. Across all different tumor entities, the PD-L1 